\title{
Two-Sided Competition of Proprietary vs. Open Source Technology Platforms and the Implications for the Software Industry
}

\author{
Nicholas Economides \\ Stern School of Business, New York University, 44 West 4th Street, New York, New York 10012, \\ neconomi@stern.nyu.edu \\ Evangelos Katsamakas \\ Graduate School of Business, Fordham University, 113 West 60th Street, New York, New York 10023, \\ katsamakas@fordham.edu
}

\begin{abstract}
$\mathrm{T}$ echnology platforms, such as Microsoft Windows, are the hubs of technology industries. We develop a framework to characterize the optimal two-sided pricing strategy of a platform firm; that is, the pricing strategy toward the direct users of the platform as well as toward firms offering applications that are complementary to the platform. We compare industry structures based on a proprietary platform (such as Windows) with those based on an open source platform (such as Linux), and analyze the structure of competition and industry implications in terms of pricing, sales, profitability, and social welfare. We find that, when the platform is proprietary, the equilibrium prices for the platform, the applications, and the platform access fee for applications may be below marginal cost, and we characterize demand conditions that lead to this. The proprietary applications sector of an industry based on an open source platform may be more profitable than the total profits of a proprietary platform industry. When users have a strong preference for application variety, the total profits of the proprietary industry are larger than the total profits of an industry based on an open source platform. The variety of applications is larger when the platform is open source. When a system based on an open source platform with an independent proprietary application competes with a proprietary system, the proprietary system is likely to dominate the open source platform industry both in terms of market share and profitability. This may explain the dominance of Microsoft in the market for PC operating systems.
\end{abstract}

Key words: networks; network effects; network externalities; complements; systems; open source software; technology platforms; software industry structure

History: Accepted by Eric von Hippel and Georg von Krogh, guest editors; received September 1, 2004. This paper was with the authors 4 months for 3 revisions.

\section{Introduction}

Technology platforms are the hubs of the value chains in technology industries. Some examples of technology platforms are Microsoft Windows (PC operating systems), Intel processors (PC hardware), and the Sony PlayStation (game consoles). The firm that becomes a platform leader and controls a platform, such as Microsoft in the operating systems market, can maintain a strong position in the industry but also faces the challenge of managing the evolution of the platform (Gawer and Cusumano 2002).

A technology platform may be proprietary or open source. A prominent example of an open source platform is Linux, an emerging operating system that is challenging the dominance of Microsoft in operating systems. The objective of this paper is to analyze the strategic differences between proprietary and open source technology platforms, and study the implications for the software industry.
A firm that controls a proprietary platform makes strategic pricing decisions for the products it sells directly to the end-user, as well as with respect to complementary products to its platform sold by other firms. We call this two-sided platform pricing. For example, firms that control game consoles set a price for the game console and often charge royalties to developers of games, following a two-sided pricing strategy. Microsoft licenses the operating system to its users, but also provides the application developers with information and resources and also makes money from licensing application development tools and support. The ability of the platform firm to use pricing strategically so as to influence the complementary goods markets has not been sufficiently analyzed in the literature.

An open source platform is used in conjunction with complementary applications that are provided by for-profit firms. An open source platform cannot 
implement a two-sided pricing strategy, but at the same time, it does not face the requirement of profitability, which may constrain the behavior of the proprietary platform firm. An emerging open source platform, such as Linux, can be used for free, but also the users face switching costs (e.g., the cost of learning, installing, and maintaining the new operating system), a smaller network of applications than the incumbent proprietary platform, and the possibility of high support costs. The applications that are complementary to an open source platform can be proprietary, and therefore have to be profitable to survive.

We develop models that extend the systems and network economics literature and provide a framework to answer the following questions. First, what is the optimal two-sided pricing strategy of a proprietary platform? Under what conditions does a platform firm set a positive or negative fee to providers of complementary applications? What are the implications of the existence of such a fee? Second, how does an industry based on a proprietary platform compare to an industry based on an open source platform with respect to prices, profitability, and social welfare? Third, what is the structure of competition between an open source platform and a proprietary platform?

A core strategic question for a platform leader is how collaborative versus competitive should its relationship be with the providers of complementary goods. Our framework provides an answer to this question in terms of access fees versus subsidies set by the platform firm. Our models allow for complementarities between the platform and each application, and at the same time, users have a preference for application variety. Thus an increase of the size and sales of the applications network increase the demand of the platform. The models also capture the exogenous switching costs incurred by the users of the open source platform.

We find that the equilibrium prices for the proprietary platform, the applications, and the platform access fee for the applications can be below marginal cost. The platform firm subsidizes the application when roughly the willingness to pay for the platform is higher than the willingness to pay for the application, or when the own-price effect of the platform is weak relative to the complementarity between the application and the platform. The platform firm subsidizes the users of the platform when the willingness to pay for the application is high relative to the willingness to pay for the platform, or the own-price effect of the application is weak relative to the complementarity between the application and the platform. The application firm subsidizes users of the application when it receives a strong subsidy by the platform firm. When more than one application is compatible to the same platform, we determine how the interaction between the applications in terms of complementarity or substitutability affects the equilibrium. We find that the platform is less likely to subsidize them if the applications are close substitutes to each other. We show that it is important to analyze the whole system of prices to determine the appropriate public policy. When the study focuses only on a part of the system, significant features of the strategic interaction in the industry are neglected or may remain unexplained.

A two-sided pricing strategy always increases the profits of the platform compared to the "one-sided" pricing strategy profits where no fees or subsidies from the platform to the application are allowed. However, the additional value to the platform of using the two-sided pricing strategy can be small depending on the market characteristics and it may not offset the costs of implementing it. We find that the two-sided pricing strategy increases the profit of the application provider only when the application is subsidized by the platform. However, implementation of a two-sided strategy often reduces social surplus and consumer surplus in the combined platform and applications markets.

We compare industry structures based on an open source and a proprietary platform. For the open source platform, we assume that it charges a zero price for the operating system as well as a zero access fee to applications, and therefore makes zero profits. The users of the platform incur a positive adoption cost, which is exogenous and captures the costs of switching to the open source platform. The applications compatible with the open source operating system can be proprietary and make positive profits. This may provide strong incentives to firms such as IBM to promote an open source platform, such as Linux, for which IBM provides proprietary applications. Indeed, we show that, under certain conditions, the profit of the application provider based on an open source platform may be larger than the total profit of the whole proprietary industry (profits of the proprietary platform plus applications). We also show that, when the cost of user switching to the open source platform is large, it is optimal for the application provider to subsidize all the users that adopt the open source platform (even though they do not all buy its application).

The social welfare comparison of industry structures based on a proprietary and an open source platform is, in general, ambiguous. However, we find that when the cost of adopting the open source platform is zero, and the platforms are of equal quality and support costs, the industry based on an open source platform has the highest social welfare. The total industry profitability is highest when the platform is proprietary and it is vertically integrated in the applications sector. 
When the platform has a network of $N>1$ independent applications, the proprietary software industry is more profitable than the industry based on an open source platform, if users have a strong preference for application variety. The variety of applications is larger when the platform is open source. This happens even though the proprietary platform subsidizes the application providers.

When a proprietary system competes with a system based on an open source platform, the proprietary system likely dominates compared to open source both in terms of market share and profitability. This is true even when the consumers' cost of adopting the open source platform is zero. This result may provide an explanation for the dominance of Microsoft in the software industry.

The structure of this paper is the following. We discuss the related literature in $\S 2$. In $\S 3$, we first analyze the equilibrium of a market with a proprietary platform with one complementary application where the platform uses a two-sided pricing strategy. Second, we analyze the market equilibrium when the platform is open source and then compare the proprietary platform with the open source platform equilibria in terms of prices, sales, consumers', and total surplus, as well as industry profitability. We then extend our results to the case of a platform with two and then with $N>2$ applications in $\S \S 4$ and 5. Finally, we analyze the competition between a proprietary platform and an open source platform in $\$ 6$. Concluding remarks and ideas for further research are in $§ 7$.

\section{Related Literature}

This paper is related to three research literature themes: (1) the economics of systems, (2) the economics of the open source software, and (3) the economics of networks and two-sided markets. The systems literature studies settings where consumers value systems composed of complementary components. The emphasis is on the implications of compatibility, the incentives of rival firms to make their components compatible, the effects of different ownership structures, and the implications of bundling. Economides and Salop (1992) study pricing in competing systems comprised of two components. Matutes and Regibeau (1988) and Economides (1989) study the incentives of firms offering such systems to make their systems compatible. Farrell and Katz (2000) show that the integration of a monopolist into a competitive complementary market may weaken the innovation incentives of independent firms. Complementary components are typically assumed to be symmetric in the systems literature. Thus, previous research has not given much attention to a setting in which the components are asymmetric and the core component (platform) sets access fees or subsidizes the other complementary components that compose the system. This paper extends the systems literature by characterizing the platform strategies in such a setting.

The literature on open source focuses mainly on the individual incentives to participate in open source projects, the incentives of firms to adopt open source initiatives, the business models of firms operating within the open source landscape, and the competitive implications of open source software (Lerner and Tirole 2005). Von Hippel and von Krogh (2003) argue that open source software development combines elements of the private and the collective innovation models. Johnson (2002) models the contribution to an open source project as a problem of private provision of a public good. Lerner and Tirole $(2001,2002)$ discuss the incentives of individual programmers and software firms to participate in open source projects. Mustonen (2003) proposes a model in which the participation of programmers in open source projects is endogenous and shows that a low implementation cost of an open source application is crucial for its survival when it competes with a proprietary application. Bitzer and Schroder (2003) consider competition in technology, rather than prices or quantities, in a software duopoly market. Mustonen (2005) analyzes when a proprietary software firm may support the development of substitute open source software.

Our paper focuses on the operating system as a platform. It departs from the above literature by considering the strategic differences between an open source and a proprietary platform and analyzing the implications for the structure of the software industry. In the literature on open source, the closest paper to this one is Casadesus and Ghemawat (2006) that studies competition between Windows and Linux. However, that paper does not consider the two-sided strategy of Windows as a platform because it takes into account only the user price of Windows and ignores the strategy of Microsoft toward the complementary applications, which is crucial in our model.

The two-sided markets and networks literature focuses on pricing strategies in platform settings such as payment systems or matching intermediaries. A market is two sided when the ratio of the platform prices matters in the equilibrium interactions between the two sides (Rochet and Tirole 2005). The platform regulates the interaction between the two sides to maximize its profit, therefore standard pricing rules may not apply to each side separately. Rochet and Tirole $(2002,2003)$ show that competition between platforms does not necessarily lead to an efficient pricing structure. The platform sets a relatively low price to the side that is valued strongly by the other side. Multihoming at one side reduces the access price 
set to the other side. Parker and Van Alstyne (2005), Caillaud and Jullien (2003), Armstrong (2006), and Hagiu (2004) analyze related determinants of pricing structure. Katsamakas and Bakos (2004) analyze platform investments in two-sided networks and the implications of different ownership structures. Overall, this literature focuses on the prices set only by the platform. Therefore prices such as the price set by application providers are abstracted away, while these prices are endogenous in our model.

Our model contributes to this literature stream by characterizing the microstructure of two-sided platform pricing in technology industries. We follow the microapproach in the networks literature (Economides 1996) and show how the cross-side network effects emerge. In the analysis that follows, we show an equivalence between a specification that assumes complementarities and a specification that assumes explicit network effects across the two sides of the market (users and application providers), thus confirming the close relationship between the two-sided networks literature and the systems literature. ${ }^{1}$

\section{The Model}

This section develops the main model of the paper analyzing a setting of a platform with one complementary application. We start with a proprietary platform and application, and continue with an open source platform with a proprietary application.

\subsection{Proprietary Platform}

Consider a setting that consists of one platform firm selling platform (for example, operating system) $A_{0}$, and an independent firm selling good $B_{1}$, which is complementary to the platform (for example, application software). The platform firm sells the platform to end-users at price $p_{0}$. The independent application provider sells the application to end-users at price $p_{1}$. The application provider also pays a per unit access fee $s$ to the platform firm (or receives a subsidy is $s$ is negative), which is set by the platform firm. A positive access fee can be interpreted as a per unit licensing fee by the operating system to applications software provider(s). A negative access fee can be interpreted as a subsidy by the platform to the application provider(s). This subsidy from the platform to the application can be created by the incorporation in the operating system of (1) functionality that is useful to the application but not directly useful to the enduser of the operating system who does not buy the application, or (2) functionality that gives additional value to the application over and above the value it

\footnotetext{
${ }^{1}$ Another distinguishing feature of our model is that we allow for users of the platform to get value even without getting access to the other side, i.e., buying applications.
}

adds to the naked operating system, or (3) functionality that reduces the costs or improves performance of the application. ${ }^{2}$

We assume a linear demand structure. The demand function of the platform $A_{0}$ is $q_{0}=a_{0}-b_{0} p_{0}-d p_{1}$, and the demand of the application $B_{1}$ is $q_{1}=a_{1}-b_{1} p_{1}-$ $d p_{0}{ }^{3}$ The quantity intercept $a_{0}$ of the platform demand (representing actual sales when all prices are zero) depends on the inherent quality and functions of the platform and the variety of applications that are compatible to the platform. The maximum sales of the platform, $a_{0}$, can be larger than the maximum sales of the application, $a_{1}$, i.e., $a_{1} \leq a_{0}{ }^{4}$ The parameter $d$ measures the strength of the complementarity between the platform and the application. We assume $b_{0}, b_{1}>$ $d$, i.e., that the own-price effect of each product dominates the cross-price effect. Finally, we assume zero costs, because marginal costs of software are close to zero and fixed costs do not affect our analysis because entry and exit are not discussed. The profit function of the platform firm is $\pi_{0}=\pi_{0 u}+\pi_{0 a}$, where $\pi_{0 u}=$ $p_{0} q_{0}$ is the platform profit from users, and $\pi_{0 a}=s q_{1}$ is the platform profit from the application access fees. The profit function of the application provider is $\pi_{1}=$ $\left(p_{1}-s\right) q_{1}$

Firms set prices in a two-stage game. In stage one, the platform sets the access fee $s$ paid by the application provider. In stage two, the platform and the application provider set prices $p_{0}, p_{1}$ simultaneously. The justification of this timing is that, first, the platform firm commits on development licensing fees or subsidies for the application provider, and then both

\footnotetext{
${ }^{2}$ For example, Microsoft is offering embedded functions to application providers, access to application programming interfaces, resources, and information for free, effectively subsidizing applications, while Microsoft is making money from application development licenses (see, e.g., developer licensing fees at http:// msdn.microsoft.com/subscriptions/licensing/default.aspx). Alternatively, we can assume that the platform firm anticipates and monitors application sales, therefore it can set access fees accordingly.

${ }^{3}$ This demand system can be generated by a population of users of differing willingness to pay. For example, it can be generated by a population of users of type $\theta \sim$ Uniform [0,1] each with a unit demand. The demand system in the text could also be generated by a representative consumer with quadratic utility function.

${ }^{4}$ This is because some platform users may not buy the application because they find the platform sufficient for their uses. For example, Windows contains a browser, an e-mail application, a media player, and word processing features, and some users may find these features sufficient and not buy any applications. The fact that some consumers may buy only the operating system and no applications is an important distinguishing feature of operating systems from other platforms such as credit card markets and markets of matching intermediaries analyzed in the existing two-sided markets literature where users are required to also buy a complementary good.
} 
firms set their prices for the users and sell their products. We assume that firms set prices noncooperatively, and we characterize the subgame-perfect Nash equilibria.

We start by analyzing the last stage of the game. The second stage necessary conditions for-profit maximization for the two firms are $\partial \pi_{0} / \partial p_{0}=\partial \pi_{1} / \partial p_{1}=0$. The best response functions of the two firms in the second stage are $p_{0}\left(p_{1}\right)=\left(a_{0}-d p_{1}-s d\right) /\left(2 b_{0}\right)$ and $p_{1}\left(p_{0}\right)=\left(a_{1}-d p_{0}+s b_{1}\right) /\left(2 b_{1}\right)$. Solving them simultaneously gives the operating system and application prices as functions of the platform access fee $s$ as $p_{0}=\left(2 a_{0} b_{1}-d a_{1}-3 d b_{1} s\right) /\left(4 b_{0} b_{1}-d^{2}\right)$ and $p_{1}=\left(2 a_{1} b_{0}-\right.$ $\left.d a_{0}+\left(2 b_{0} b_{1}+d^{2}\right) s\right) /\left(4 b_{0} b_{1}-d^{2}\right)$. Notice that $d p_{1} / d s>0$ and $d p_{0} / d s<0$; that is, as expected, the application price increases with the platform fee $s$ because the application firm faces a higher marginal cost, while the platform price decreases as the application has a higher price. These two effects imply that sales of the operating system (respectively, application) increase (decrease) in the platform fee $s$ :

$$
\begin{gathered}
\frac{d q_{0}}{d s}=-b_{0} \frac{d p_{0}}{d s}-d \frac{d p_{1}}{d s}>0 \quad \text { and } \\
\frac{d q_{1}}{d s}=-b_{1} \frac{d p_{1}}{d s}-d \frac{d p_{0}}{d s}<0 .
\end{gathered}
$$

In the first stage of the game, the platform firm chooses fee $s$, anticipating the second-stage equilibrium prices. Its necessary condition for profit maximization is

$$
\frac{d \pi_{0}}{d s}=\left(p_{0} \frac{d q_{0}}{d s}+q_{0} \frac{d p_{0}}{d s}\right)+\left(s \frac{d q_{1}}{d s}+q_{1}\right)=0 .
$$

A marginal increase of $s$ affects both profit streams of the platform firm. The profit from users increases by $p_{0} d q_{0} / d s$ and decreases by $q_{0}\left|d p_{0} / d s\right|$. The profit from the application firm increases by $q_{1}$ and decreases by $s\left|d q_{1} / d s\right|$. Both profit streams of the platform are concave (inverted U-shaped) in $s$, and therefore the total platform profit is concave is $s$. The platform's choice of $s$ maximizes the sum of the two profit streams. The effect of $s$ on the platform profit from users is

$$
\begin{aligned}
& \frac{d \pi_{0 u}(s)}{d s} \\
& =d \frac{d\left(a_{1}\left(2 b_{0} b_{1}+d^{2}\right)-6 b_{1}\left(b_{0} b_{1}-d^{2}\right) s\right)-2 a_{0} b_{1}\left(2 b_{0} b_{1}+d^{2}\right)}{\left(4 b_{0} b_{1}-d^{2}\right)^{2}} .
\end{aligned}
$$

The profit from users is decreasing at $s=0$, since

$$
\frac{d \pi_{0 u}(0)}{d s}=d \frac{a_{1} d\left(2 b_{0} b_{1}+d^{2}\right)-2 a_{0} b_{1}\left(2 b_{0} b_{1}+d^{2}\right)}{\left(4 b_{0} b_{1}-d^{2}\right)^{2}}<0 .
$$

Therefore the fee $s_{u}^{*}$ that would maximize only the platform profit from users is negative.

\section{Figure 1 Platform Profit Streams and Access Fee $s^{*}$}

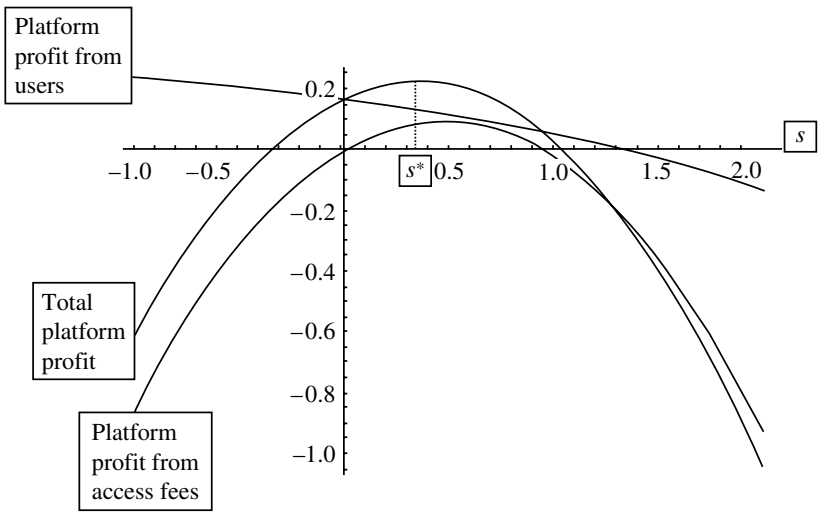

The effect of fee $s$ on the platform profit from the application is

$$
\frac{d \pi_{0 a}(s)}{d s}=b_{1} \frac{2 a_{1} b_{0}-a_{0} d-4\left(b_{0} b_{1}-d^{2}\right) s}{4 b_{0} b_{1}-d^{2}} .
$$

This profit is increasing at $s=0$ if $2 a_{1} b_{0}-a_{0} d>0$. Then, $s_{a}^{*}$ is positive, and therefore $s^{*}$ may be positive or negative $\left(s_{u}^{*}<s^{*}<s_{a}^{*}\right)$. The access fee $s^{*}$ is positive when at $s=0$ the platform profit from the application is increasing at a faster rate than the profit from users is decreasing. Figure 1 shows an example of that case. Figure 2 shows the relationship between the platform's access fee, the platform profit, the application's profit, and the total industry surplus.

The two-stage game has a unique subgame-perfect Nash equilibrium given by the following prices:

$$
\begin{gathered}
s^{*}=\frac{a_{1}\left(8 b_{0}^{2} b_{1}^{2}+d^{4}\right)-a_{0} b_{1} d\left(8 b_{0} b_{1}+d^{2}\right)}{2 b_{1}\left(b_{0} b_{1}-d^{2}\right)\left(8 b_{0} b_{1}+d^{2}\right)}, \\
p_{0}^{*}=\frac{a_{0} b_{1}\left(8 b_{0} b_{1}+d^{2}\right)-a_{1} d\left(10 b_{0} b_{1}-d^{2}\right)}{2\left(b_{0} b_{1}-d^{2}\right)\left(8 b_{0} b_{1}+d^{2}\right)}, \\
p_{1}^{*}=\frac{a_{1}\left(12 b_{0}^{2} b_{1}^{2}-2 b_{0} b_{1} d^{2}-d^{4}\right)-a_{0} b_{1} d\left(8 b_{0} b_{1}+d^{2}\right)}{2 b_{1}\left(b_{0} b_{1}-d^{2}\right)\left(8 b_{0} b_{1}+d^{2}\right)} .
\end{gathered}
$$

Figure 2 Profits and Total Surplus

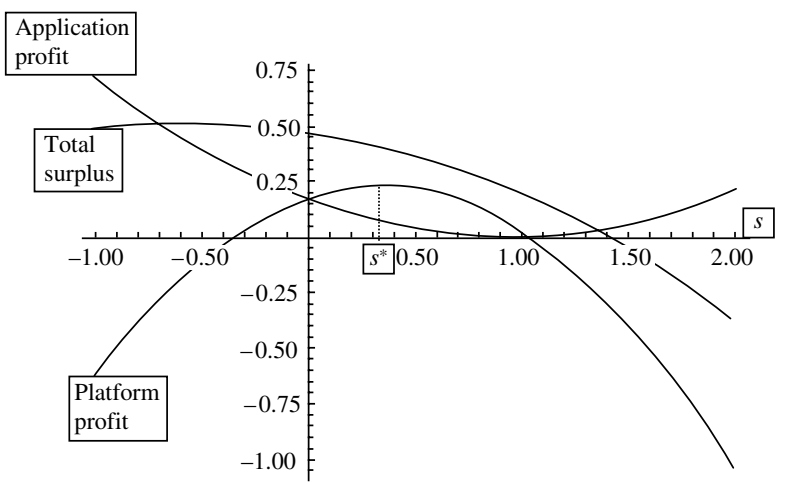


3.1.1. Equivalent Formulation with Cross-Component Network Effects. We can rewrite the demand system in an alternative but equivalent formulation with explicit cross-component network effects, which will be useful in interpreting the results

$$
q_{0}=\alpha_{0}-\beta_{0} p_{0}+\varepsilon_{0} q_{1}, \quad q_{1}=\alpha_{1}-\beta_{1} p_{1}+\varepsilon_{1} q_{0} .
$$

In this setup, sales of each good depend on its own price as well as on a "network effect" arising from sales of a complementary good. The cross-component network effects are defined as $\varepsilon_{0} \equiv \partial q_{0} / \partial q_{1}, \varepsilon_{1} \equiv$ $\partial q_{1} / \partial q_{0}$. The marginal network effect coefficient $\varepsilon_{0}$ captures the increase in the demand of the platform by a marginal increase of the application sales. In short, $\varepsilon_{0}$ is the network effect of applications sales on platform sales. Similarly, marginal network effect coefficient $\varepsilon_{1}$ captures the increase in the demand for the application by a marginal increase in platform sales. In short, $\varepsilon_{1}$ is the network effect of platform sales on applications sales. From the initial assumptions, we have $0<\varepsilon_{0}, \varepsilon_{1}<1$. The network effects $\varepsilon_{0}$, $\varepsilon_{1}$ are useful in characterizing and interpreting the results. To see the equivalence of the two demand formulations, we invert the original demand system, and identify the coefficients as

$$
\alpha_{0} \equiv\left(a_{0} b_{1}-a_{1} d\right) / b_{1}, \quad \alpha_{1} \equiv\left(a_{1} b_{0}-a_{0} d\right) / b_{0} ，
$$

and

$$
\beta_{0} \equiv\left(b_{0} b_{1}-d^{2}\right) / b_{1}, \quad \beta_{1} \equiv\left(b_{0} b_{1}-d^{2}\right) / b_{0}
$$

and the explicit marginal cross-component network effects as $\varepsilon_{0} \equiv d / b_{1}, \varepsilon_{1} \equiv d / b_{0}$.

3.1.2. Subsidization Conditions. An important issue in the two-sided markets literature is when a platform may subsidize the one or the other side of the two-sided market (see, for example, Armstrong 2006). There have also been frequent allegations of below-cost platform pricing with anticompetitive intent. Below, we characterize the conditions under which the equilibrium prices are below cost, i.e., negative in our setup of zero costs.

Access Fee s. An increase of $s$ from $s=0$ increases the profit that the platform firm captures from application access fees, but decreases the profit that the platform firm captures from sales of the platform to users. When the first effect dominates, the platform sets a positive $s^{*}$, and, when the second effect dominates, $s^{*}$ is negative. The access fee $s^{*}$ is negative if and only if

$$
\frac{a_{0}}{a_{1}} \geq \frac{8+\left(\varepsilon_{0} \varepsilon_{1}\right)^{2}}{\varepsilon_{1}\left(8+\varepsilon_{0} \varepsilon_{1}\right)} .
$$

Therefore, the larger $a_{0} / a_{1}$, the more likely it is that the platform firm subsidizes the application provider.
Figure 3 The Region Where $s^{*}<0$

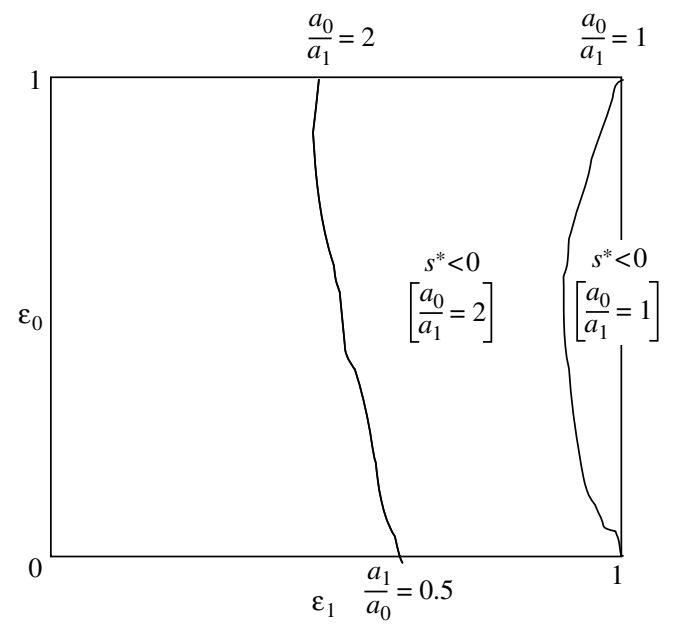

Essentially, this means that the platform will subsidize the application when the maximum sales of application is relatively small so that the platform will reap the network effect from the additional sales of the application. ${ }^{5}$ Figure 3 shows the regions where $s^{*}$ is negative for two different levels of $a_{0} / a_{1}$. In the region where the platform provider chooses a negative $s^{*}, \varepsilon_{1}$ is strong, which is implied by a strong crossprice effect $(d)$ relative to the own-price effect $\left(b_{0}\right)$. When the platform firm subsidizes the application firm, the application firm sets a low or negative application price and this shifts the demand of the platform up given that the cross-price effect is strong. This shift combined with the weak own-price effect $b_{0}$ enable the platform firm to set a high price $p_{0}$ and capture a high profit from the platform users.

Platform Price $p_{0}$. The platform firm sets a negative application fee $p_{0}$ when this allows the platform firm to set a large application access fee. The platform firm subsidizes the users of the platform if an increase of profit from an associated increase of access fees imposed on the application provider offsets the decrease of profit from end-users. The platform price $p_{0}^{*}$ is negative if and only if

$$
\frac{a_{0}}{a_{1}} \leq \varepsilon_{0} \frac{10-\varepsilon_{0} \varepsilon_{1}}{8+\varepsilon_{0} \varepsilon_{1}} .
$$

Thus the platform price is negative when the demand for the application is relatively strong compared to the demand for the platform $\left(a_{0} / a_{1}\right.$ is small) and $\varepsilon_{0}$ is strong (cross-price effect $d$ strong relative to the ownprice effect of the application $b_{1}$ ). Then, a negative

\footnotetext{
${ }^{5}$ Note that the negative access fee of the platform is not an indication of predatory pricing, and occurs naturally in a monopoly setting under no threat of entry, and it is not any entry-prevention strategy.
} 
platform price combined with a strong complementarity (large $d$ ) shifts the application demand upward. The strong application demand (strong $a_{1}$ ) combined with a weak $b_{1}$ enables the application provider to set a high price $p_{1}^{*}$ and the platform firm to set a high access fee to the application providers.

Application Price $p_{1}$. The application firm (which is independent of the platform) sets a negative application price when its cost of subsidizing the users is lower than the per unit subsidy it collects from the platform firm. The application price is negative if and only if

$$
\frac{a_{0}}{a_{1}} \geq \frac{12-2 \varepsilon_{0} \varepsilon_{1}-\left(\varepsilon_{0} \varepsilon_{1}\right)^{2}}{\varepsilon_{1}\left(8+\varepsilon_{0} \varepsilon_{1}\right)} .
$$

This condition is more restrictive than the condition for a negative $s^{*}$, which means that the application price is set below marginal cost only when the application receives a subsidy from the platform. Similar to the condition for a negative $s^{*}$, it is necessary that the platform has a larger demand than the application (large $a_{0} / a_{1}$ ), or that $\varepsilon_{1}$ is strong, which is implied by the own-price effect of the application being weak relative to the cross-price effect.

3.1.3. The Effects of Using a Two-Sided Pricing Strategy. A platform firm can easily follow a "onesided" pricing strategy (by setting only an end-user price and $s=0$ ) rather than a two-sided strategy. A two-sided pricing strategy can be costly to implement (for example, to implement the two-sided strategy the platform firm needs to keep track of the sales of the application providers). To see the effects of using a two-sided strategy, we define the additional value $V_{0}$ created by the two-sided strategy for the platform firm as the platform's profit from the two-sided strategy minus its profit from the onesided strategy, $V_{0}=\pi_{0}^{*}-\left.\pi_{0}^{*}\right|_{s=0}$. The benefit or loss of the two-sided strategy for the application provider is defined similarly: $V_{1}=\pi_{1}^{*}-\left.\pi_{1}^{*}\right|_{s=0}$.

The two-sided strategy does not affect the equilibrium sales and profits when the platform and the application are one-to-one (perfect) complements, i.e., when $d=b_{0}=b_{1}=b$ and $a_{0}=a_{1}=a$. Then, both the platform and the application firm face the same system demand $q=a-b\left(p_{0}+p_{1}\right)$. In this case, when the platform sets $s$, there is an infinity of Nash price equilibria of the form $\left\langle p_{0}^{*}=a /(3 b)-s, p_{1}^{*}=a /(3 b)+s, s\right\rangle$. At each of these equilibria, sales $q_{0}^{*}=q_{1}^{*}=a / 3$, and the equilibrium profit is $\pi_{0}^{*}=\pi_{1}^{*}=a^{2} /(3 b)$. Therefore, in this special case, the access fee does not matter. Thus, in our model, the access fee $s$ is effectively a device for the platform firm to discriminate between the users who buy the system (platform and application) and the users who buy only the platform. When the system demand is small because of a small application demand, then a negative access fee $s^{*}$ enables the platform firm essentially to set a high platform price $p_{0}^{*}$ for end-users who buy only the platform and effectively a low price $p_{0}^{*}+s^{*}$ for end-users who buy the system. When the system demand is strong, a positive $s^{*}$ enables the platform firm essentially to set a low platform price $p_{0}^{*}$ for end-users who buy only the platform and effectively a high price $p_{0}^{*}+s^{*}$ for end-users who buy the system.

To analyze the effect of the two-sided strategy, we need to characterize the equilibrium when the platform follows a one-sided pricing strategy, i.e., when $s=0$ and the first stage of the game is eliminated. The profit function of the platform firm is then $\pi_{0}=p_{0} q_{0}$ and the profit function of the application provider is $\pi_{1}=p_{1} q_{1}$. The two-stage game with the restriction of $s=0$ has a unique Nash equilibrium at prices

$$
p_{0}^{*}=\frac{2 a_{0} b_{1}-a_{1} d}{4 b_{0} b_{1}-d^{2}}, \quad p_{1}^{*}=\frac{2 a_{1} b_{0}-a_{0} d}{4 b_{0} b_{1}-d^{2}} .
$$

Evaluating the effect of using a two-sided strategy, we have, as expected that if there are no costs of implementing the two-sided strategy, the platform firm is at least as well off implementing it, i.e., $V_{0} \geq 0$ since the one-sided strategy is a restriction of the two-sided strategy at $s=0$. The effect of the twosided strategy on the platform value is always positive but U-shaped in $a_{0} / a_{1}$. Therefore it might not be profitable for a platform firm to implement such a strategy when implementation is very costly. We also find that using a two-sided strategy benefits the application firm $\left(V_{1} \geq 0\right)$ if and only if the platform firm subsidizes the application firm. ${ }^{6}$ Consumers' and total surplus may increase or decrease when the platform firm uses a two-sided strategy rather than a one-sided strategy.

\subsection{Open Source Platform}

We now consider an open source platform $A_{0}$ and a complementary application $B_{1}$, with demand functions $q_{0}=a_{0}^{O}-b_{0} c_{u}-d p_{1}$ and $q_{1}=a_{1}-b_{1} p_{1}-d c_{u}$, respectively. We assume that the maximum demand $a_{0}^{O}$ of the open source platform is, in general, different than the maximum demand $a_{0}$ of the proprietary platform, but all the other demand parameters $a_{1}, b_{0}, b_{1}, d$ are identical for both platforms. We also assume that the open source platform sets a zero user price and zero access fees to application(s), i.e., $p_{0}=s=0$. Thus the open source platform profits are zero. However, the complementary application $\left(B_{1}\right)$ can set a positive price to users. We assume that there is an exogenous user cost $c_{u}$ of platform adoption, representing the

${ }^{6}$ The effect $V_{1}$ of the two-sided strategy on the application firm is positive if $\left(\left(\varepsilon_{0} \varepsilon_{1}\right)^{2}+8-\left(a_{0} / a_{1}\right) \varepsilon_{1}\left(8+\varepsilon_{0} \varepsilon_{1}\right)\right)\left(\left(\varepsilon_{0} \varepsilon_{1}\right)^{2}-4 \varepsilon_{0} \varepsilon_{1}-24+\right.$ $\left.\left(a_{0} / a_{1}\right) \varepsilon_{1}\left(8+\varepsilon_{0} \varepsilon_{1}\right)\right)>0$. The second parenthesis is negative, and the first parenthesis is negative if and only if $s^{*}$ is negative. 
cost of installing and learning the open source operating system. This exogenous cost is assumed to be zero for the proprietary platform. Thus, in the static setting of our model, $c_{u}$ captures the switching cost to the open source platform.

The profit functions for the open source platform and the proprietary application are $\pi_{0}=0$ and $\pi_{1}=$ $p_{1} q_{1}$. The equilibrium price for the application in the open source software industry is $p_{1}^{*}=\left(a_{1}-d c_{u}\right) /\left(2 b_{1}\right)$. The firms' sales and profits at equilibrium are

$$
\begin{gathered}
q_{0}^{*}=\frac{2 a_{0}^{O} b_{1}-a_{1} d-\left(2 b_{0} b_{1}-d^{2}\right) c_{u}}{2 b_{1}}, \quad q_{1}^{*}=\frac{a_{1}-d c_{u}}{2}, \\
\pi_{1}^{*}=\frac{\left(a_{1}-d c_{u}\right)^{2}}{4 b_{1}} .
\end{gathered}
$$

Notice that the open source industry has positive sales, and therefore the equilibrium exists only if the cost $c_{u}$ is relatively small. ${ }^{7}$ When $c_{u}$ is relatively large, i.e., $c_{u}>\left(a_{0}^{O} b_{1}-a_{1} d\right) /\left(b_{0} b_{1}-d^{2}\right)$, the application provider may also increase its own profits by giving all the users of the open source platform a subsidy $t$, therefore reducing the effective platform adoption cost to $c_{u}-t .^{8}$

\subsection{Vertically Integrated Proprietary Platform}

A proprietary software industry may be vertically integrated. For example, Microsoft produces both Windows and Office. Vertical integration is not possible in an open source industry because the operating system is not proprietary. The profit-maximizing prices under a vertically integrated proprietary software industry are

$$
p_{0}^{*}=\frac{a_{0} b_{1}-a_{1} d}{2\left(b_{0} b_{1}-d^{2}\right)} \quad \text { and } \quad p_{1}^{*}=\frac{a_{1} b_{0}-a_{0} d}{2\left(b_{0} b_{1}-d^{2}\right)} .
$$

The application price is below marginal cost (in our setup, negative) if and only if $\varepsilon_{1}=d / b_{0}<a_{1} / a_{0}$. Therefore the integrated firm sells the application below marginal cost when the network effect of platform sales on applications sales $\varepsilon_{1}$ is sufficiently weak or (equivalently) when there is a relatively strong demand for the operating system compared to applications resulting in a much larger demand intercept of the platform $a_{0}$ than the demand intercept of the application $a_{1}$. Then, the extra profit that the firm makes from selling the platform offsets the cost of subsidizing the use of the application.

\footnotetext{
${ }^{7}$ In particular, $q_{1}^{*}>0 \Leftrightarrow c_{u}<a_{1} / d$ and $q_{0}^{*}>0 \Leftrightarrow c_{u}<\left(2 a_{0} b_{1}-a_{1} d\right) /$ $\left(2 b_{0} b_{1}-d^{2}\right)$.

${ }^{8}$ The analysis of this subsidization case is available from the authors upon request. An example of this would be IBM, a provider of proprietary applications for Linux subsidizing Linux.
}

\subsection{Comparison of Industry Structures}

We now compare the three industry structures (vertically integrated proprietary, vertically disintegrated proprietary, and open source platform with proprietary applications) in terms of profits and social surplus.

Proposition 1. Among the three industry structures, vertically integrated proprietary, vertically disintegrated proprietary, and open source platform with proprietary applications, total industry profits are highest for the vertically integrated proprietary industry structure.

PRoof. The total profit of the vertically integrated industry minus the profit of the open source industry is $\left(a_{1} d-a_{0} b_{1}\right)^{2} /\left(4 b_{1}\left(b_{0} b_{1}-d^{2}\right)\right)>0$. The total profit of the vertically integrated industry minus the total profit of a vertically disintegrated proprietary industry is

$$
\frac{a_{1}^{2}\left(16 b_{0}^{2} b_{1}^{2}-4 d^{2} b_{0} b_{1}-3 d^{4}\right)}{4 b_{1}\left(8 b_{0} b_{1}+d^{2}\right)^{2}}>0 .
$$

Intuitively, the vertically integrated industry is the most profitable industry structure because it internalizes the complementarity (vertical externality) between the platform and the application. The vertically integrated monopolist can always replicate the prices of the other two structures, therefore it can never be less profitable than them.

As Proposition 2 shows, the comparison between the total profits of the industry based on the open source platform and the total profits of the proprietary platform industry with independent firms is ambiguous.

PRoposition 2. The proprietary software industry with independent firms is more profitable than the industry based on an open source platform if and only if

$$
\begin{aligned}
\frac{\varepsilon_{1}}{\varepsilon_{0}}(8 & \left.+\varepsilon_{0} \varepsilon_{1}\right)^{2} x^{2}-2 \varepsilon_{1}\left(8+\varepsilon_{0} \varepsilon_{1}\right)^{2} x-16+84 \varepsilon_{0} \varepsilon_{1} \\
& +15\left(\varepsilon_{0} \varepsilon_{1}\right)^{2}-2\left(\varepsilon_{0} \varepsilon_{1}\right)^{3} \geq 0
\end{aligned}
$$

where $x=a_{0} / a_{1}$ is the relative sales for the platform relative to the application realized at zero prices for all products.

An increase of the demand $a_{0}$ for the proprietary platform increases the profitability of the proprietary industry. ${ }^{9}$ Therefore, when $a_{0} / a_{1}$ is large, the proprietary industry is more profitable than the industry based on the open source platform. Conversely, an industry based on the open source platform can be

\footnotetext{
${ }^{9}$ But notice that the demand intercept $a_{0}^{O}$ for the open source platform does not affect the profitability of the industry based on the open source platform (because the open source platform charges a zero price irrespective of the size of the demand), and therefore does not appear in the condition of Proposition 2.
} 
more profitable than the proprietary industry when $a_{0} / a_{1}$ is relatively small, $\varepsilon_{1}$ is relatively small, and $\varepsilon_{0}$ is relatively large. This means that for a given complementarity $d$, the own-price effect $b_{0}$ of the platform is large and the own-price effect $b_{1}$ of the application is small. Under these conditions, the application provider's profit is large and this strengthens the relative profitability of the industry that is based on an open source platform.

The comparison of social surplus across the three industry structures is, in general, ambiguous. Proposition 3 describes the comparison of social surplus across the three industry structures, when $c_{u}$ is small and the platforms have identical quality, i.e., $a_{0}=a_{0}^{O}$.

Proposition 3. Among the three industry structures (vertically integrated proprietary, vertically disintegrated proprietary, and open source platform with proprietary applications), the open source industry is the structure with the largest total welfare if the cost of open source adoption $c_{u}$ is small.

Proof is by direct calculation. Intuitively, the industry based on the open source platform dominates the other two industry structures, because the zero platform price leads to higher platform and application adoption.

\section{Platform with Two Applications}

\subsection{Proprietary Platform with Two Applications}

We now extend the main model to the case of a platform with two applications. The two applications remain complements to the platform but can be either substitutes or complements to each other. We start with the analysis of a proprietary platform.

The demand function of the platform $A_{0}$ is $q_{0}=a_{0}-$ $b_{0} p_{0}-\sum d p_{i}$, and the demand of application $B_{i}$ is $q_{i}=$ $a_{i}-b_{i} p_{i}-d p_{0}+c p_{j}$, where $i, j=1,2$. Parameter $c$ measures the degree of substitution between applications $i$ and $j$. We assume symmetric applications' demands, $a_{2}=a_{1}, b_{2}=b_{1}$, as well as $a_{1}<a_{0}$ and $b_{0}>2 d, b_{1}>d$, $b_{1}>|c|, b_{1}>d+c$. The profit functions for platform and applications are $\pi_{0}=p_{0} q_{0}+s \sum q_{i}$ and $\pi_{i}=\left(p_{i}-\right.$ s) $q_{i}, i=1,2$.

As in the earlier models, the platform sets fees to applications in the first stage of a game and both platform and applications set user fees in the second stage. Because of symmetric demands, the platform firm sets the same access price $s$ for each application. The necessary conditions for profit maximization by the platform and the applications' firms in the second stage are $\partial \pi_{0} / \partial p_{0}=\partial \pi_{i} / \partial p_{i}=0$. The best response functions of the firms are

$$
p_{0}\left(p_{1}\right)=\frac{a_{0}-2 d p_{1}-s d}{2 b_{0}} \text { and } p_{1}\left(p_{0}\right)=\frac{a_{1}-d p_{0}+s b_{1}}{2 b_{1}-c}
$$

which give

$$
p_{0}=\frac{a_{0}\left(2 b_{1}-c\right)-2 d a_{1}-2 d s\left(3 b_{1}-c\right)}{2\left(b_{0}\left(2 b_{1}-c\right)-d^{2}\right)}
$$

and

$$
p_{1}=\frac{2 a_{1} b_{0}-d a_{0}+\left(2 b_{0} b_{1}+d^{2}\right) s}{2\left(b_{0}\left(2 b_{1}-c\right)-d^{2}\right)} .
$$

Notice that $d p_{1} / d s>0$ and $d p_{0} / d s<0$. The first-stage condition for profit maximization of the platform is

$$
\frac{d \pi_{0}}{d s}=\left(p_{0} \frac{d q_{0}}{d s}+q_{0} \frac{d p_{0}}{d s}\right)+\sum\left(s \frac{d q_{i}}{d s}+q_{i}\right)=0 .
$$

The platform profit from users is decreasing at $s=0$, since

$$
\frac{d \pi_{0 u}(0)}{d s}=d \frac{2 d a_{1}\left(b_{0} b_{1}+d^{2}\right)-a_{0}\left(2 b_{1}-c\right)\left(b_{0} b_{1}+d^{2}\right)}{\left(b_{0}\left(2 b_{1}-c\right)-d^{2}\right)^{2}}<0 .
$$

The platform profit from the application access fees is increasing at $s=0$, since

$$
\frac{d \pi_{0 a}(0)}{d s}=b_{1} \frac{2 a_{1} b_{0}-a_{0} d}{b_{0}\left(2 b_{1}-c\right)-d^{2}}>0
$$

following from the assumption $2 a_{1} b_{0}-a_{0} d>0$. There is a unique subgame-perfect Nash equilibrium $\left\langle s^{*}, p_{0}^{*}, p_{i}^{*}\right\rangle$ given by the following:

$$
\begin{aligned}
s^{*}= & \left(2 a_{1}\left(b_{0}^{2} b_{1}\left(2 b_{1}-c\right)+d^{4}\right)\right. \\
& \left.-a_{0} d\left(2 b_{0} b_{1}\left(2 b_{1}-c\right)+d^{2}\left(b_{1}-c\right)\right)\right) \\
& \cdot\left(2 \left(2 b_{0}^{2} b_{1}\left(2 b_{1}^{2}-3 b_{1} c+c^{2}\right)+b_{0} d^{2}\left(-7 b_{1}^{2}+2 b_{1} c+c^{2}\right)\right.\right. \\
& \left.\left.+2 d^{4}\left(-b_{1}+c\right)\right)\right)^{-1} \\
p_{0}^{*}= & \left(a_{0}\left(b_{1}-c\right)\left(2 b_{0} b_{1}\left(2 b_{1}-c\right)+d^{2}\left(b_{1}-c\right)\right)\right. \\
& \left.+2 a_{1} d\left(b_{0} b_{1}\left(-5 b_{1}+3 c\right)+d^{2}\left(b_{1}+c\right)\right)\right) \\
& \cdot\left(2 \left(2 b_{0}^{2} b_{1}\left(2 b_{1}^{2}-3 b_{1} c+c^{2}\right)+b_{0} d^{2}\left(-7 b_{1}^{2}+2 b_{1} c+c^{2}\right)\right.\right. \\
& \left.\left.+2 d^{4}\left(-b_{1}+c\right)\right)\right)^{-1} \\
p_{i}^{*}= & \left(a_{0} d\left(2 b_{0} b_{1}\left(-2 b_{1}+c\right)+d^{2}\left(-b_{1}+c\right)\right)\right. \\
& \left.+a_{1}\left(2 b_{0}^{2} b_{1}\left(3 b_{1}-2 c\right)-2 b_{0} d^{2}\left(b_{1}+c\right)-2 d^{4}\right)\right) \\
& \cdot\left(2 \left(2 b_{0}^{2} b_{1}\left(2 b_{1}^{2}-3 b_{1} c+c^{2}\right)+b_{0} d^{2}\left(-7 b_{1}^{2}+2 b_{1} c+c^{2}\right)\right.\right. \\
& \left.\left.+2 d^{4}\left(-b_{1}+c\right)\right)\right)^{-1} .
\end{aligned}
$$

The access fee $s^{*}$ is negative if and only if

$$
\frac{a_{0}}{a_{1}} \geq \frac{2\left(2-\zeta_{1}\right)+\left(\varepsilon_{0} \varepsilon_{1}\right)^{2}}{\varepsilon_{1}\left(2\left(2-\zeta_{1}\right)+\varepsilon_{0} \varepsilon_{1}\left(1-\zeta_{1}\right)\right)},
$$

where $\zeta_{1}=c / b_{1}$. The right-hand side of this condition is increasing in $\zeta_{1}$. Therefore the platform is unlikely to subsidize the applications when the competitive effect between the applications is strong. Similarly, 


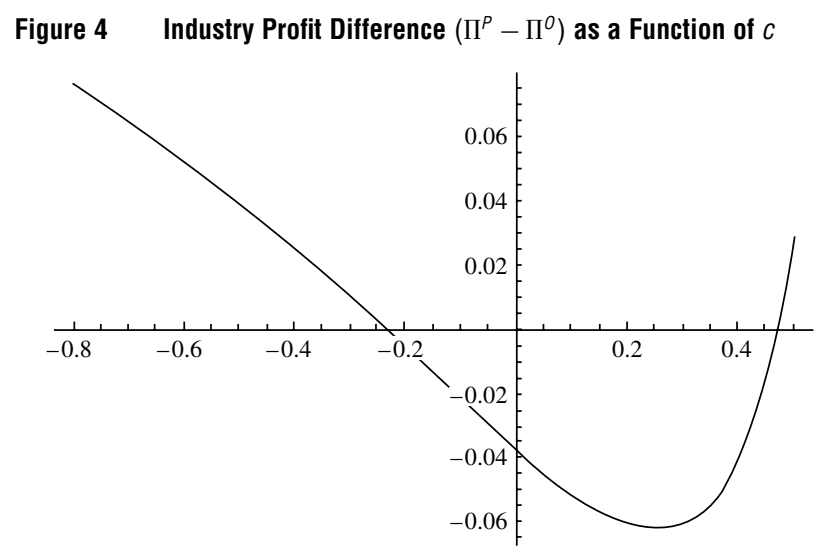

the platform is likely to subsidize the applications when the applications are complementary to each other (e.g., a spreadsheet application and a presentation application). ${ }^{10}$

\subsection{Open Source Platform with Two Applications} When the platform is open source and the two complementary applications are directly sold to endusers by independent for-profit firms, the equilibrium prices, sales, and profits are

$$
\begin{gathered}
s^{*}=0, \quad p_{0}^{*}=0, \quad p_{i}^{*}=\frac{a_{1}}{2 b_{1}-c}, \\
q_{0}^{*}=\frac{a_{0}^{O}\left(2 b_{1}-c\right)-2 a_{1} d}{2 b_{1}-c}, \quad q_{1}^{*}=\frac{a_{1} b_{1}}{2 b_{1}-c}, \quad \pi_{0}^{*}=0, \\
\pi_{i}^{*}=\frac{a_{1}^{2} b_{1}}{\left(2 b_{1}-c\right)^{2}} .
\end{gathered}
$$

\subsection{Comparison of Industry Structures}

The following proposition compares the profitability of the industry based on open source and proprietary platforms.

Proposition 4. When there are two for-profit applications, the proprietary software industry is more profitable than the industry based on an open source platform in terms of total industry profits if and only if

$$
\begin{aligned}
x^{2}[ & \left.\left(b_{1}-c\right)\left(2 b_{1}-c\right)^{2}\left(2 b_{0} b_{1}\left(2 b_{1}-c\right)+d^{2}\left(b_{1}-c\right)\right)^{2}\right] \\
& -x\left[4 d\left(2 b_{1}-c\right)^{2}\left(2 b_{0} b_{1}\left(2 b_{1}-c\right)+d^{2}\left(b_{1}-c\right)\right)^{2}\right] \\
& +b_{0}^{2} b_{1}^{2} d^{2}\left(-84 b_{1}^{3}+96 b_{1}^{2} c-29 b_{1} c^{2}+c^{3}\right) \\
& +2 b_{0} b_{1} d^{4}\left(-15 b_{1}^{3}+33 b_{1}^{2} c-17 b_{1} c^{2}+2 c^{3}\right) \\
& +d^{6}\left(8 b_{1}^{3}-5 b_{1} c^{2}+c^{3}\right)>0,
\end{aligned}
$$

where $x=a_{0} / a_{1}$.

\footnotetext{
${ }^{10}$ Moreover, the platform price $p_{0}$ is negative if and only if $a_{0} / a_{1} \leq$ $2 \varepsilon_{0}\left(\left(5-3 \zeta_{1}\right)-\left(1+\zeta_{1}\right) \varepsilon_{0} \varepsilon_{1}\right) /\left(\left(1-\zeta_{1}\right)\left(2\left(2-\zeta_{1}\right)+\left(1-\zeta_{1}\right) \varepsilon_{0} \varepsilon_{1}\right)\right)$; that is, generally when the intercept of the platform demand is small compared to applications.
}

The proprietary industry is more profitable when $x$ is large. This happens because an increase of $a_{0}$ increases the proprietary industry profit, but $\alpha_{0}^{O}$ does not affect the profit of the applications that are compatible with the open source platform.

When $x$ is relatively small, the industry based on an open source platform can be more profitable than the proprietary industry. This occurs when $|c|$ is relatively small. Figure 4 shows an example of the relative profitability of the two industry structures as a function of $c$, for $x=1.1, b_{0}=1, b_{1}=1, d=0.3$.

Proposition 4 shows that the complementarity and substitutability between the applications affects the relative profitability of the industry based on a proprietary and the industry based on an open source platform.

\section{Platform with $N$ Independent Applications}

\subsection{Proprietary Platform}

This section generalizes the main model to $N$ applications that are independent from each other (that is, neither substitutes nor complements with each other). We start with the analysis of a proprietary platform. Let the demand function for the platform $A_{0}$ be $q_{0}=$ $a_{0}-b_{0} p_{0}-\sum d_{i} p_{i}$, and the demand for the application $B_{i}$ be $q_{i}=a_{i}-b_{i} p_{i}-d_{i} p_{0}+\sum c_{i j} p_{j}$, where $i, j=$ $1, \ldots, N$. We assume $d_{i}<b_{i}, \sum d_{i}<b_{0}$, all applications' demands are symmetric, $a_{i}=a_{1}, b_{i}=b_{1}, d_{i}=d$ (and therefore $N d<b_{0}$ ), and because each application is independent of other applications, $c_{i j}=0, j=$ $1, \ldots, N-1$. The profit functions are $\pi_{0}=p_{0} q_{0}+s \sum q_{i}$ and $\pi_{i}=\left(p_{i}-s\right) q_{i}, i=1, \ldots, N$.

The quantity intercept of the demand for the platform (realized at zero prices $p_{0}=p_{i}=0$ ) can be defined as $a_{0} \equiv A+E(N)$, where $A$ is the standalone demand for the platform, and $E(N)$ an increasing and concave function that captures the additional value to consumers created by adding a variety of applications.

Adding a new application $i$ has the following additional network effect on platform demand: $[E(N)-$ $E(N-1)]+\varepsilon_{0 i}$, where $\varepsilon_{0 i}=d_{i} / b_{i}$. In this equation, the first bracket captures the increase in the platform demand because of an increase of the variety of applications. This increase in the platform demand is significant when the users have a strong preference for application variety. The second term captures the effect of a marginal increase in the demand of the new application. Thus the users value the platform based on the variety of platform applications and the actual adoption of each application.

The second-stage necessary conditions for profit maximization of the platform and the applications 
are $\partial \pi_{0} / \partial p_{0}=\partial \pi_{i} / \partial p_{i}=0, i=1, \ldots, N{ }^{11}$ Because of symmetry among applications, $p_{i}=p_{1}, i=1, \ldots, N$. The best response function of an application is not affected by $N$, since $p_{1}\left(p_{0}\right)=\left(a_{1}-d p_{0}+b_{1} s\right) /\left(2 b_{1}\right)$. The best response of the platform firm, $p_{0}\left(p_{1}\right)=\left(a_{0}-\right.$ $\left.N d p_{1}-N d s\right) /\left(2 b_{0}\right)$, is impacted by two competing shifts as $N$ increases: (1) it shifts upward because of the increase of application variety and (2) it shifts downward because the effect of the application price on the platform demand is scaled with $N$ and the lost profit from the application side is also scaled with $N$. The first effect dominates when users value the application variety strongly.

The first-stage profit maximization condition is $d \pi_{0} / d s=0$. The effect of fee $s$ on the platform profit from the applications is scaled with $N$. The platform profit from users at $s=0$,

$$
\frac{d \pi_{0 u}(0)}{d s}=N d \frac{\left(2 b_{0} b_{1}+N d^{2}\right)\left(N d a_{1}-2 b_{1} a_{0}\right)}{\left(4 b_{0} b_{1}-N d^{2}\right)^{2}},
$$

is decreasing only when $N d a_{1} /\left(2 b_{1}\right)<a_{0}$.

The symmetric subgame perfect Nash equilibrium is

$$
\begin{gathered}
s^{*}=\frac{a_{1}\left(8 b_{0}^{2} b_{1}^{2}+N^{2} d^{4}\right)-a_{0} b_{1} d\left(8 b_{0} b_{1}+N d^{2}\right)}{2 b_{1}\left(b_{0} b_{1}-N d^{2}\right)\left(8 b_{0} b_{1}+N d^{2}\right)}, \\
p_{0}^{*}=\frac{a_{0} b_{1}\left(8 b_{0} b_{1}+N d^{2}\right)-N a_{1} d\left(10 b_{0} b_{1}-N d^{2}\right)}{2\left(b_{0} b_{1}-N d^{2}\right)\left(8 b_{0} b_{1}+N d^{2}\right)}, \\
p_{1}^{*}=\frac{a_{1}\left(12 b_{0}^{2} b_{1}^{2}-2 b_{0} b_{1} N d^{2}-N^{2} d^{4}\right)-a_{0} b_{1} d\left(8 b_{0} b_{1}+N d^{2}\right)}{2 b_{1}\left(b_{0} b_{1}-N d^{2}\right)\left(8 b_{0} b_{1}+N d^{2}\right)} .
\end{gathered}
$$

As the size $N$ of the network of applications of the platform increases, the profit of the platform and each application increases, i.e., $d \pi_{i}^{*} / d N>0$ and $d \pi_{0}^{*} / d N>$ 0 . The application provider profit $\pi_{i}^{*}$ is not affected by a change in the users preference for variety because $\pi_{i}^{*}$ is independent of $a_{0}$.

We now determine the relationship between the fee or subsidy $s^{*}$ and the size of the applications network $N$. When users have a strong preference for applications variety, the access fee $s^{*}$ is decreasing in $N$, and, for large $N$, it becomes negative. Then, the addition of a new application in the network benefits the other applications too, because they either pay a lower access fee or they receive a larger subsidy. When users have a strong preference for variety, $a_{0}$ increases fast as $N$ increases. Each application sets a low price because it faces a low access cost (or is subsidized). Thus, there is a strong demand for the

$$
\begin{aligned}
& { }^{11} \text { These can be written as } \\
& \qquad \frac{\partial \pi_{0}}{\partial p_{0}}=q_{0}+p_{0} \frac{\partial q_{0}}{\partial p_{0}}+s \sum_{i=1}^{N} \frac{\partial q_{i}}{\partial p_{0}}=\left(a_{0}-2 b_{0} p_{0}-d \sum_{i=1}^{N} p_{i}\right)-N s d=0, \\
& \frac{\partial \pi_{i}}{\partial p_{i}}=q_{i}+\left(p_{i}-s\right) \frac{\partial q_{i}}{\partial p_{i}}=\left(a_{1}-2 b_{1} p_{i}-d p_{0}\right)+b_{1} s=0, \quad i=1, \ldots, N .
\end{aligned}
$$

platform, which enables the platform firm to capture most of its profit from platform sales. However, if users have a weak preference for application variety, the access fee $s^{*}$ is increasing in $N$. Then, the platform captures most of its profit from the application providers. Finally, if users have a moderate preference for variety, $s^{*}$ is initially decreasing, and then increasing in $N$.

The increase of the application network size $N$ has a similar effect on $p_{1}^{*}$. However, a larger $N$ has the opposite effect on the platform price $p_{0}^{*}$. When the users have a strong preference for variety, $d p_{0}^{*} / d N>$ 0 . When the users have a weak preference for variety, $d p_{0}^{*} / d N<0$ and $p_{0}^{*}$ become negative for large $N$. The distribution of the industry profit between the platform and the applications $\left(\pi_{0}^{*} /\left(N \pi_{1}^{*}\right)\right)$ is U-shaped in $N$ when users assign small value to application variety, and monotonically increasing in $N$ when users value strongly variety in applications.

Summarizing, the crucial feature in this setting is that, as the number of applications increases, the platform firm faces the trade off of capturing more profits either from the application providers fees or from the application users. When users value application variety strongly, it is likely that the second effect dominates and the platform firm subsidizes the application providers and makes its profits from the end-users.

\subsection{Open Source Platform}

Assume that the platform is open source and $N$ applications are independently produced by for-profit firms. The demand for the platform $A_{0}$ is $q_{0}=a_{0}^{O}-$ $\sum d_{i} p_{i}$, and the demand of the application $B_{i}$ is $q_{i}=$ $a_{i}-b_{i} p_{i}$, where $i, j=1, \ldots, N$. The profit functions of the application firms are $\pi_{i}=p_{i} q_{i}, i=1, \ldots, N$. We assume symmetric applications' demands, i.e., for $a_{i}=a_{1}, b_{i}=b_{1}, d_{i}=d$, and as before $N d<b_{0}$. Platform sets $p_{o}=0$ and competition among application providers leads to the unique Nash equilibrium set of prices $p_{i}^{*}=a_{1} /\left(2 b_{1}\right)$. Equilibrium sales are $q_{0}^{*}=$ $\left(2 a_{0}^{O} b_{1}-a_{1} d N\right) /\left(2 b_{1}\right)$ and $q_{i}^{*}=a_{1} / 2$. Each application provider makes profit $\pi_{i}^{*}=a_{1}^{2} /\left(4 b_{1}\right)$. The total industry profit is $\Pi^{O}=N a_{1}^{2} /\left(4 b_{1}\right)$.

\subsection{Variety of Applications}

The total profit when the industry is based on an open source platform is independent of the intercept $a_{0}^{O}$ of the platform demand because the open source platform charges a zero price irrespective of the demand. Therefore the total profit does not depend on the users preference for application variety, which affects only $a_{0}^{O}$. However, the total profit of the proprietary industry is increasing on $a_{0}$, which is increasing in the application variety $N$. This observation leads us to the following comparison of industry profits between an industry based on a proprietary platform and one based on an open source platform. 
Proposition 5. The proprietary industry is more profitable than the industry based on an open source platform, when the users' preference for variety is strong.

Proof. The total profit difference between the proprietary and the open source industry structures is

$$
\begin{aligned}
\Pi^{P}-\Pi^{O} \geq 0 \Leftrightarrow & x^{2}\left(N^{2} \varepsilon_{0} \varepsilon_{1}^{3}+16 N \varepsilon_{1}^{2}+64 \frac{\varepsilon_{1}}{\varepsilon_{0}}\right) \\
& -x\left(32 N^{2} \varepsilon_{0} \varepsilon_{1}^{2}+128 N \varepsilon_{1}+2 N^{3} \varepsilon_{0}^{2} \varepsilon_{1}^{3}\right) \\
& +84 N^{2} \varepsilon_{0} \varepsilon_{1}+15 N^{3} \varepsilon_{0}^{2} \varepsilon_{1}^{2} \\
& -16 N-2 N^{4} \varepsilon_{0}^{3} \varepsilon_{1}^{3} \\
\geq & 0,
\end{aligned}
$$

where $x=a_{0} / a_{1}$.

The industry based on an open source platform cannot internalize the users preference for variety into profits. In contrast, the proprietary platform is able to internalize the preference for variety and this leads to higher industry profits. The proposition implies that the stronger the preference for variety, the larger the reduction of the industry profits from a potential shift of the software industry to an open source platform.

We now extend the model, allowing for free entry of applications in a proprietary and open source platforms. We introduce another stage at the beginning of the game, where each application firm decides whether to enter the industry. There is a population of $\bar{N}$ application providers ( $\bar{N}$ large), each offering one application if it decides to enter the industry. The fixed cost of entry is a random variable $k$ distributed according to c.d.f. $G(k)$ over $[0, K]$, where $K$ is large. Then, a necessary and sufficient condition for firm $i$ to enter the industry is $k_{i} \leq \pi_{i}^{*}$. Because, in our model, the applications are symmetric and $\pi_{i}^{*}$ is the same for all $i$, the number of applications at equilibrium will be $\bar{N} G\left(\pi_{i}^{*}\right)$.

The equilibrium profits for an open source application when there are $N$ independent applications are $\pi_{i}^{O *}=a_{1}^{2} /\left(4 b_{1}\right)$, while the equilibrium profits for an application when the platform is proprietary are

$$
\pi_{1}^{P *}=\frac{a_{1}^{2}\left(2 b_{0} b_{1}+N d^{2}\right)^{2}}{b_{1}\left(8 b_{0} b_{1}+N d^{2}\right)^{2}} .
$$

Direct calculation shows that $\pi_{1}^{P *}<\pi_{i}^{O *}$ for all $N$. Therefore, at the free entry equilibrium, there will be larger application variety under an open platform. ${ }^{12}$

Proposition 6. The profits of an application provider are higher under an open source platform. Therefore the free entry application variety $N^{P}$ of an industry based on a proprietary platform is smaller than the application variety $N^{O}$ of an industry based on an open source platform.

$12 \sqrt{\pi_{1}^{P *} / \pi_{i}^{O *}}=\left(4 b_{0} b_{1}+2 N d^{2}\right) /\left(8 b_{0} b_{1}+N d^{2}\right)<1 \Leftrightarrow N d^{2}<4 b_{0} b_{1} \Leftarrow$ $b_{0} d<4 b_{0} b_{1} \Leftarrow d<b_{1}$ since $N d<b_{0}$.
Even though the proprietary platform subsidizes the application providers when $N$ is large and the application provider profit is increasing in $N$, still the application providers are better off when the platform is open source. The zero price of the open source platform leads to high platform adoption and higher willingness to pay for the complementary applications. As a result of the larger number of applications for the open source platform $\left(N^{O}>N^{P}\right)$, the social welfare of the industry based on an open source platform will be larger than in a proprietary industry.

\section{Competition Between Systems Based on a Proprietary and an Open Source Platform}

Consider now platforms $A_{1}, A_{2}$, where $A_{1}$ is a proprietary platform and $A_{2}$ is an open source platform and proprietary applications $B_{1}, B_{2}$, and assume that platform $A_{i}$ is compatible only with application $B_{i}$. We call the pair $\left(A_{1}, B_{1}\right)$ the proprietary system, and the pair $\left(A_{2}, B_{2}\right)$ the open source system. The demand function of platform $i$ is $q_{A_{i}}=$ $a_{i}-b_{i} p_{A_{i}}-d p_{B_{i}}+e\left(p_{A_{j}}+p_{B_{j}}\right)$ and the demand of the application $i$ is $q_{B_{i}}=a_{i}-b_{i} p_{B_{i}}-d p_{A_{i}}+e\left(p_{A_{j}}+p_{B_{i}}\right), i=$ 1,2 . The parameter $e>0$ captures the degree of substitution between the two systems. As we did earlier, we assume that $b_{i}>e$ so that own-price effects dominate cross-price effects. The two platforms have potentially different demand intercepts $a_{1}, a_{2}$, which depend on the number of features bundled with each platform, the inherent quality of the platform, and the size of the network of applications compatible with the platform. Since platform $A_{2}$ is open source, $p_{A_{2}}=$ $s_{2}=0$ and the exogenous adoption cost of the platform is $c_{u}$. For expositional clarity, we assume $b_{1}=$ $b_{2}=d=1$, i.e., that the platform and the application are perfect complements. Firms play a two-stage game, where access fee $s$ is imposed on the application provider at the first stage, and end-user prices are determined in the second stage.

We first analyze the case when the proprietary platform and the application are produced by different firms. The profit functions are $\pi_{A_{1}}=p_{A_{1}} q_{A_{1}}+s_{1} q_{B_{1}}$, $\pi_{B_{1}}=\left(p_{B_{1}}-s_{1}\right) q_{B_{1}}$, and $\pi_{B_{2}}=p_{B_{2}} q_{B_{2}}$. The second-stage profit maximization conditions are $\partial \pi_{A_{1}} / \partial p_{A_{1}}=0$, $\partial \pi_{B_{i}} / \partial p_{B_{i}}=0, i=1,2$. The first-stage profit maximization condition is $\partial \pi_{A_{1}} / \partial s_{1}=0$. The equilibrium sales, prices, and profits are

$$
\begin{gathered}
q_{A_{1}}^{*}=q_{B_{1}}^{*}=\frac{p_{A_{1}}^{*}+p_{B_{1}}^{*}}{2}=\frac{2 a_{1}+e\left(a_{2}+c_{u}\right)}{2\left(3-e^{2}\right)}, \\
q_{A_{2}}^{*}=q_{B_{2}}^{*}=p_{B_{2}}^{*}=\frac{2 a_{1} e+3 a_{2}-c_{u}\left(3-2 e^{2}\right)}{2\left(3-e^{2}\right)}, \\
p_{A_{2}}^{*}=0, \quad \text { and } \pi_{A_{1}}^{*}=\pi_{B_{1}}^{*}=p_{A_{1}}^{* 2}, \quad \pi_{A_{2}}^{*}=0, \quad \pi_{B_{2}}^{*}=p_{B_{2}}^{* 2} .
\end{gathered}
$$


We focus on the effect of $a_{i}, e$, and $c_{u}$ on the adoption and profitability of the two competing systems. The adoption (sales) of the proprietary platform is larger than the adoption of the open source platform when

$$
\frac{a_{2}(3-e)-2 a_{1}(1-e)}{3+e-2 e^{2}}<c_{u} \cdot{ }^{13}
$$

This implies that, generally, for a relatively large demand for the proprietary application (large $a_{1}$ ), a large transition cost for the open platform (large $c_{u}$ ), and a relatively small demand for application of the open source platform $\left(a_{2}\right.$ small $)$, the proprietary platform will dominate in terms of market share. For $a_{2} / a_{1}<$ $2(1-e) /(3-e)$, the proprietary platform dominates for all nonnegative switching costs. The right-hand side is positive and decreasing in $e$; therefore for the inequality to hold, a larger divergence of maximum sales under the two platform settings is required for closer substitute systems. When the systems are far substitutes ( $e$ close to 0 ) and the maximum demand for the open source platform is less than two-thirds of the maximum demand for the proprietary platform, the proprietary platform dominates even when the adoption cost of the platform $c_{u}$ is zero. In the other extreme, when the systems are very close substitutes (e close to 1 ), the open source platform has higher sales unless $c_{u}$ is very large $\left(a_{2}<c_{u}\right)$.

In the special case of equal maximum demands for the open source and the proprietary platforms, $a_{2}=$ $a_{1}=a$, the proprietary platform has a larger market share when $a(1+e) /\left(3+e-2 e^{2}\right)<c_{u}$. The left-hand side is increasing in the degree of substitution $e$ so that a higher and higher switching cost $c_{u}$ is necessary for the proprietary platform to dominate as the platforms are closer and closer substitutes.

Total industry profits are higher for the vertically disintegrated proprietary system compared to the system based on an open source platform when

$$
\frac{a_{2}(3-e \sqrt{2})-2 a_{1}(\sqrt{2}-e)}{3+e \sqrt{2}-2 e^{2}}<c_{u} \cdot{ }^{14}
$$

This implies that, generally, for a relatively large demand for the proprietary application (large $a_{1}$ ), a large transition cost for the open platform (large $c_{u}$ ), and a relatively small demand for application of the open source platform $\left(a_{2}\right.$ small), the proprietary system will dominate in terms of profits. For $a_{2} / a_{1}<$ $2(\sqrt{2}-e) /(3-e \sqrt{2})$, the proprietary system dominates in terms of profits for all nonnegative switching

\footnotetext{
${ }^{13}$ All the parentheses in this equation and the denominator are positive since $e \leq 1$.

${ }^{14} \pi_{A_{1}}^{*}+\pi_{B_{1}}^{*}-\left(\pi_{A_{2}}^{*}+\pi_{B_{2}}^{*}\right)=2 p_{A_{1}}^{* 2}-p_{B_{2}}^{* 2}=\left(p_{A_{1}}^{*} \sqrt{2}-p_{B_{2}}^{*}\right)\left(p_{A_{1}}^{*} \sqrt{2}+p_{B_{2}}^{*}\right)>$ $0 \Leftarrow p_{A_{1}}^{*} \sqrt{2}<p_{B_{2}}^{*} \Leftrightarrow\left(a_{2}(3-e)-2 a_{1}(1-e)\right) \cdot\left(3+e-2 e^{2}\right)^{-1}<c_{u}$. Again, all the parentheses in this equation and the denominator are positive since $e \leq 1$.
}

costs. The right-hand side is positive and decreasing in $e$; therefore for the inequality to hold, a larger divergence of maximum sales under the two platform settings is required for closer substitute systems. When the systems are far substitutes ( $e$ close to 0 ) and the maximum demand for the open source platform is less than $(2 / 3) \sqrt{2} \cong 0.93$ of the maximum demand for the proprietary platform, the proprietary system dominates in terms of profits even when the adoption cost of the platform $c_{u}$ is zero.

In the special case of equal maximum demands for the open source and the proprietary platforms, $a_{2}=$ $a_{1}=a$, the proprietary platform industry has larger profits when

$$
\frac{a(3-2 \sqrt{2})+e(2-\sqrt{2})}{3+e \sqrt{2}-2 e^{2}}<c_{u} .
$$

The left-hand side is increasing in the degree of substitution $e$ so that a higher and higher switching cost $c_{u}$ is necessary for the proprietary platform to dominate as the platforms are closer and closer substitutes.

Proposition 7. A vertically disintegrated proprietary system dominates a competing system based on an open source platform in terms of market share and profits provided that the maximum demand of the open source system $\left(a_{2}\right)$ is relatively small compared to the maximum demand of the proprietary system $\left(a_{1}\right)$. When the substitution between the systems is low and/or when the switching $\operatorname{cost} c_{u}$ is high, the proprietary system is more likely to dominate.

When a vertically integrated proprietary system competes against a system of an open source platform combined with a proprietary application, the profit functions are $\pi_{1}=p_{A_{1}} q_{A_{1}}+p_{B_{1}} q_{B_{1}}, \pi_{A_{2}}=0$, and $\pi_{B_{2}}=$ $p_{B_{2}} q_{B_{2}}$. The noncooperative equilibrium prices, sales, and profits are

$$
\begin{gathered}
q_{A_{1}}^{*}=q_{B_{1}}^{*}=p_{A_{1}}^{*}+p_{B_{1}}^{*}=\frac{2 a_{1}+e\left(a_{2}+c_{u}\right)}{4-e^{2}}, \quad p_{A_{2}}^{*}=0, \\
\pi_{1}^{*}=q_{A_{1}}^{* 2}, \quad \pi_{A_{2}}^{*}=0, \quad \pi_{B_{2}}^{*}=q_{B_{2}}^{* 2},
\end{gathered}
$$

and

$$
q_{A_{2}}^{*}=q_{B_{2}}^{*}=p_{B_{2}}^{*}=\frac{a_{1} e+2 a_{2}-c_{u}\left(2-e^{2}\right)}{4-e^{2}} .
$$

The vertically integrated proprietary system has a larger market share than the system based on an open source platform if $\left(a_{2}-a_{1}\right)(2-e) /\left(2+e-e^{2}\right)<c_{u} \cdot{ }^{15}$ Therefore the vertically integrated platform dominates the open source platform in terms of sales for a relatively large demand for the proprietary application (large $a_{1}$ ), a large transition cost for the open

${ }^{15}$ The second parenthesis in the numerator and the denominator are positive. 
platform (large $c_{u}$ ), and a relatively small demand for application of the open source platform $\left(a_{2}\right.$ small). When the maximum sales of the system based on the open source platform (realized at all zero prices) are smaller than the maximum sales of the vertically integrated proprietary system, $a_{1}>a_{2}$, the proprietary system dominates for all nonnegative switching costs.

Total profits in each system are proportional to platform sales in that structure, $\pi_{A_{1}}^{*}+\pi_{B_{1}}^{*}=q_{A_{1}}^{* 2}, \pi_{A_{2}}^{*}+$ $\pi_{B_{2}}^{*}=q_{B_{2}}^{* 2}$. Therefore the comparison between system profits is exactly the same as the comparison between system sales.

Proposition 8. A vertically integrated system dominates a competing system based on an open source platform both in terms of profitability and market share, unless the maximum demand of the open source system $\left(a_{2}\right)$ (realized at all zero prices) is larger than the maximum demand of the proprietary system $\left(a_{1}\right)$.

Propositions 7 and 8 provide an explanation for the dominance of the Microsoft in the operating systems market. Microsoft controls also significant applications such as the Office productivity suite. This vertical integration, along with the fact that the demand for Windows is larger than the demand for Linux $\left(a_{1}>a_{2}\right)$ (because of the larger number of applications compatible with Windows), enables Microsoft to coordinate the provision of the platform and its applications through appropriate pricing that internalizes the network effects. The proposition shows that this will be true even when the switching cost to Linux is zero.

\section{Concluding Remarks}

We developed models that analyze the strategic differences between a proprietary technology platform such as Microsoft Windows, and an open source platform such as Linux. We show that it is important to evaluate competition not just in the platform market or just in the applications markets, but additionally in the combined interaction across these markets. We show that the introduction of a third price, a fee that application developers may pay or receive from the platform (and one that is used extensively in practice) makes a very significant difference in firms' competitive interactions and the evaluation of the platform applications competitive landscape from the point of view of public policy.

In our setup, the proprietary platform firm can set positive prices to the end-users of the platform or, alternatively, subsidize them. The platform firm can also set positive access fees to the providers of applications that are compatible with the platform, or, alternatively, subsidize these providers. When using both the price to its end-users as well as the access fee to the platform provider, the proprietary platform firm follows a two-sided pricing strategy to maximize its profit. We analyzed this strategy and characterized its effects compared to a "one-sided" platform strategy that sets only end-user prices.

When, alternatively, the platform is open source, we assume that the platform is provided for free to endusers as well as to application providers. In the open source platform setting, the application providers sell their applications at a positive price and make profits, and can also subsidize the adoption of the open source platform when that increases their profit.

When the platform is proprietary, we found that the equilibrium prices for the platform, or the applications, and the platform access fee for the applications can be below marginal cost without this being a predatory strategy. It follows that it is important to analyze the whole system of prices to determine the appropriate public policy in technology industries. When the study focuses only on a part of the system, significant features of the strategic interaction in the industry are neglected or may remain unexplained.

When more than one application uses the same platform, we have shown how the degree of substitutability among the applications affects the equilibrium. We show that a proprietary platform is less likely to subsidize the applications if the substitutability between the applications is strong and more likely to subsidize them if the applications are complementary to each other. When the platform has a network of $N>1$ independent applications, then the platform profit and each application profit is increasing in $N$. The access fee paid by each application to the platform is decreasing in $N$ only when the users have a strong preference for application variety.

We compared a software industry based on a proprietary platform with a software industry based on an open source platform. We found that a vertically integrated industry is more profitable than both an open source platform industry as well as a vertically disintegrated proprietary industry. However, the open source industry is more profitable than the vertically disintegrated proprietary platform industry when the demand of the proprietary platform is not much stronger than the demand of the application, the platform users have a weak preference for application variety, and the own-price effect of the platform is strong, while the own-price effect of the application is weak. Conversely, the vertically disintegrated proprietary industry is more profitable than the open source platform industry when the demand for the proprietary platform is significantly larger than the demand of the application, the own-price effect of the platform is weak, while the own-price effect of the application is strong. We also found that the variety of applications is larger when the platform is open source. 
When a proprietary system competes with a system based on an open source platform, then the proprietary system most likely dominates both in terms of market share and profitability. This holds even when the cost of adopting the open source platform is zero.

Future research in this area may include a study of the determination of quality in the context of an open source platform, an understanding of the incentive to innovate under open source (both for the platform and the applications), and a comparison with the incentive to innovate in a proprietary platform setting, and more generally, the dynamics of competition in a multiperiod setting.

\section{Acknowledgments}

This paper evolved from a chapter of the second coauthor's doctoral dissertation at Stern Business School, New York University. The authors contributed equally and the names appear in alphabetical order. We thank the editors, reviewers, and the participants at Telecommunications Policy Research Conference 2005 for their comments.

\section{References}

Armstrong, M. 2006. Competition in two-sided markets. RAND J. Econom. Forthcoming.

Bitzer, J., P. Schroder. 2003. Competition and innovation in a technology setting software duopoly. Discussion Paper 363, Deutsches Institut für Wirtschaftsforschung, Berlin, Germany.

Caillaud, B., B. Jullien. 2003. Chicken and egg: Competing matchmakers. RAND J. Econom. 34(2) 309-328.

Casadesus-Masanell, R., P. Ghemawat. 2006. Dynamic mixed duopoly: A model motivated by Linux vs. Windows. Management Sci. 52(7) 1072-1084.

Economides, N. 1989. Desirability of compatibility in the absence of network externalities. Amer. Econom. Rev. 79(5) 1165-1181.

Economides, N. 1996. The economics of networks. Internat. J. Indust. Organ. 14(6) 673-699.
Economides, N., S. C. Salop. 1992. Competition and integration among complements, and network market structure. J. Indust. Econom. 40(1) 105-123.

Farrell, J., M. L. Katz. 2000. Innovation, rent extraction, and integration in systems markets. J. Indust. Econom. 48(4) 413-432.

Gawer, A., M. A. Cusumano. 2002. Platform Leadership. Harvard Business School Press, Boston, MA.

Hagiu, A. 2004. Optimal pricing and commitment in two-sided markets. Working paper, Princeton University, Princeton, NJ.

Johnson, J. P. 2002. Open source software: Private provision of a public good. J. Econom. Management Strategy 11(4) 637-662.

Katsamakas, E., Y. Bakos. 2005. Design and ownership of two-sided networks. J. Econom. Perspectives 19(2) 99-120.

Lerner, J., J. Tirole. 2001. The open source movement: Key research questions. Eur. Econom. Rev. 45(4-6) 819-826.

Lerner, J., J. Tirole. 2002. Some simple economics of open source. J. Indust. Econom. 50(2) 197-234.

Lerner, J., J. Tirole. 2005. The economics of technology sharing: Open source and beyond. J. Econom. Perspectives 19(2) 99-120.

Matutes, C., P. Regibeau. 1988. Mix and match: Product compatibility without network externalities. RAND J. Econom. 19(Summer) 221-234.

Mustonen, M. 2003. Copyleft-The economics of Linux and other open source software. Inform. Econom. Policy 15(1) 99-121.

Mustonen, M. 2005. When does a firm support substitute open source programming. J. Econom. Management Strategy 14(1) 121-139.

Parker, G., M. Van Alstyne. 2005. Two-sided network effects: A theory of information product design. Management Sci. 51(10) 1494-1504.

Rochet, J. C., J. Tirole. 2002. Cooperation among competitors: Some economics of payment card associations. RAND J. Econom. 33(4) 549-570.

Rochet, J. C., J. Tirole. 2003. Platform competition in two-sided markets. J. Eur. Econom. Assoc. 1(4) 990-1029.

Rochet, J. C., J. Tirole. 2005. Two-sided markets. Toulouse Conf. TwoSided Markets. Working paper, Institut d'Economie Industrielle, Toulouse, France.

von Hippel, E., G. von Krogh. 2003. Open source software and the "private-collective" innovation model. Organ. Sci. 14(2) 209-223. 